Research Paper

\title{
HPV58 E7 Protein Expression Profile in Cervical Cancer and CIN with Immunohistochemistry
}

\author{
Qiaoli Zheng"1, Xianzhen chen1, Rui Han¹, Jiang Zhu1, Hui Wang², Lingjing Chen, Yinjing Song1, Luxia \\ Chen $^{1}$, Hao Cheng ${ }^{1 凶}$ and Na Jin ${ }^{1 凶}$ \\ 1. Department of Dermatology, Sir Run Run Shaw Hospital, School of Medicine, Zhejiang University, Hangzhou, Zhejiang Province, China. \\ 2. Department of Pathology, The First People's Hospital of Fuyang, Hangzhou, Zhejiang Province, China. \\ 3. Department of Dermatology, Hangzhou Children's Hospital, Hangzhou, Zhejiang Province, China. \\ $\triangle$ Corresponding authors: Hao Cheng, E-mail: chenghao1@zju.edu.cn; Tel: +86-571-86006976; Fax: +86-571-86044817; Address: 3 East Qingchun Road, Hangzhou, Zhejiang, \\ China, 310016. Na Jin, E-mail:3202039@zju.edu.cn; Tel: +86-571-86006975; Fax: +86-571-86044817; Address: 3 East Qingchun Road, Hangzhou, Zhejiang, China, 310016. \\ (c) The author(s). This is an open access article distributed under the terms of the Creative Commons Attribution License (https://creativecommons.org/licenses/by/4.0/). \\ See http://ivyspring.com/terms for full terms and conditions.
}

Received: 2020.07.18; Accepted: 2020.12.15; Published: 2021.01.18

\begin{abstract}
Background: The persistent infection of high-risk human papillomavirus (HR-HPV) is one of the most common causes of cervical cancer worldwide, and HPV type 58 (HPV58) is the third most common HPV type in eastern Asia. The E7 oncoprotein is constitutively expressed in HPV58-associated cervical cancer cells and plays a key role during tumorigenesis. This study aimed to assess the HPV58 E7 protein expression in the tissues of cervical cancer and cervical intraepithelial neoplasia (CIN).

Methods: A total of 67 HPV58-positive cervical samples were collected, including 25 cervical cancer samples and $42 \mathrm{CIN}$ samples. All the tissues were examined by HPV58 E7, p16INK4a and Ki67 immunohistochemistry $(\mathrm{IHC})$. At last, we analyzed their association with clinical and pathological variables.

Results: HPV58 E7 expression was detected in $96 \%$ of the HPV58 DNA-positive cervical cancer tissues and 85.7\% of HPV58-positive CIN tissues. 65 samples of cervical cancer and CIN tissues had p16-positive staining, while 59 samples were $\mathrm{Ki}-67$ positive.

Conclusions: HPV58 E7 protein is highly expressed in both cervical cancer and CIN tissues. HPV58 E7 IHC could be sensitive and specific for evaluating HPV-driven cervical cancer and pre-cancerous lesions, in combination with p16 and $\mathrm{Ki}-67 \mathrm{IHC}$.
\end{abstract}

Key words: cervical cancer; CIN; E7; HR-HPV; immunohistochemistry

\section{Introduction}

Cervical cancer is one of the most common female genital cancers [1]. High-risk human papillomavirus (HR-HPV) is one major cause of cervical cancer, which accounts for over 500,000 new cancer cases and over 250,000 cancer deaths per year [2]. HPV type $16(50 \%)$ and $18(15 \%)$ are the most common subtypes worldwide. However, HPV type 58 (HPV58) plays a more prominent role in cervical cancer and pre-cancer (cervical intraepithelial neoplasia 1 (CIN1), CIN2, CIN3) in Asian countries. It is identified in $11.5 \%$ to $28 \%$ of cervical cancer patients in China [3].

HPVs are small, nonenveloped double-stranded DNA viruses that replicate their genomes in the nuclei of host cells (the keratinocytes). HR-HPV infection leads to the activation of viral gene expression, including the early genes E6 and E7 [4]. Their expression is important for cellular transformation process. The E7 oncoproteins degrade and inactivate the retinoblastoma tumor suppressor protein $\mathrm{pRB}$, contributing to the malignant transformation of the host cells [5, 6]. Specifically, the genomic and transcriptomic profiles of HPV58 in cervical neoplasia have been studied, and variants such as T20I and G63S substitutions at E7 of HPV58 were proposed to confer a significantly higher risk for cervical neoplasia $[7,8]$. However, the proteomes of HPV58, especially E7 oncoprotein, has not been well addressed. The detection of HPV58 E7 protein could be critical both in establishing the diagnosis of malignancy and assigning an HPV-related etiology to malignancy. 
To date, a commercial antibody to HPV58 E7 for immunohistochemistry detection is still unavailable. We have produced and verified the specific polyclonal antibody against the HPV58 E7 protein [9]. In this study, we aimed to detect the HPV58 E7 protein expression using this antibody in the tissues of HPV58-positive cervical cancer and CIN with IHC. Meanwhile, we detected the Ki-67 and p16 expression, analyzed the correlations between HPV58 E7, p16, or Ki-67 expression levels and clinical and pathological variables. The E7 oncoproteins expression would be a potential marker for HPV58-related cervical cancer and CIN diagnosis and disease progression.

\section{Methods}

\section{Study population}

This study was performed according to the Declaration of Helsinki. The study protocol was approved by the Ethics Committee of Sir Run Run Shaw Hospital of Zhejiang University School of Medicine, China.

The following inclusion criteria were required to be included in the study: (1) histologically confirmed diagnosis of cervical cancer or CIN; (2) positive result of the HPV58 DNA test. Exclusion criteria included previous treatment for the cervical disease (including loop electrosurgical excision procedure (LEEP), cold-knife conization, cryotherapy, LASER therapy, or hysterectomy, prior chemotherapy or radiation treatment for cervical neoplasia, pregnancy, HIV infection, previous or co-existence of other malignant diseases. 25 cervical cancer patients and $42 \mathrm{CIN}$ patients who were HPV58-positive detected by polymerase chain reaction (PCR) were included between January 2009 and August 2016 from Sir Run Run Shaw Hospital of Zhejiang University School of Medicine. Written informed consent was obtained from all the participants. Formalin-fixed and paraffin-embedded (FFPE) specimens from cervical cancer patients and CIN patients diagnosed according to the WHO classification of tumors of female reproductive organs by the Division of Pathology and were collected by the Departments of Obstetrics and Gynecology, Sir Run Run Shaw Hospital. Authors could access information that could identify individual participants during and after data collection.

\section{Patient evaluation and disease monitoring}

All patients' routine history had been taken, a physical examination, HPV test and cervical biopsy for morphological analysis. Morphologic diagnoses were based on consensus review by 2 subspecialty gynecologic pathologists. When morphology was equivocal, the diagnoses were adjudicated by a third gynecologic pathologist. LEEP or hysterectomy was performed and the removed tissues were transferred for histopathological examination. Besides, we collected the data on the degree of cell differentiation, tumor size and lymph node metastasis. Cervical carcinoma or CIN recurrences would be detectable by cervical biopsy during follow-up visits.

\section{HPV genotyping assay}

Total DNA was extracted from $200 \mu$ l exfoliated cervical cell suspension with the HPV DNA extraction kit (TELLGEN, Shanghai, China) according to the manufacturer's instructions. Using the HPV DNA genotyping kit (TELLGEN, Shanghai, China), the Luminex ${ }^{\circledR}$ xMAPTM technology can quickly and quantitatively detect 27 types of HPV (high risk subtypes 16, 18, 31, 33, 35, 39, 45, 51, 52, $56,58,59$, 66, $68,26,53,82$, and low risk subtypes $6,11,40,42,43$, $44,55,61,81,83)$. Briefly, $5 \mu \mathrm{l}$ extracted DNA solution was used for PCR in a reaction $(20 \mu \mathrm{l})$ containing $10 \mu \mathrm{l}$ PCR mixture, $5 \mu \mathrm{l}$ primer mixture, and $0.8 \mu \mathrm{l}$ rTaq polymerase. The PCR amplification was performed and $3 \mu \mathrm{l}$ products were added to microsphere hybrid for hybridization procedure on an ABI9700 (ThermoFisher, Waltham, U.S.A.). Each reaction pore was added $75 \mu \mathrm{LA}$ S-PE. The mixture was detected on Luminex200 multifunctional flow detector (R\&D system, Minneapolis, U.S.A.).

\section{Immunohistochemistry analysis}

FFPE HPV58-positive (detected by PCR) cervical cancer or CIN sections $(8-\mu \mathrm{m})$ were used for immunohistochemical staining of the HPV58-E7 protein [9], anti-p16 mouse monoclonal antibody (mAb) (1:500, GT201302, Gene, Shanghai, China) and anti-Ki-67 mouse mAb (1:500, MAB-0672, MXB, Fuzhou, China). Endogenous peroxidases in the de-paraffinized sections were quenched with $0.3 \%$ hydrogen peroxide in $60 \%$ methanol for $20 \mathrm{~min}$. Nonspecific adsorption was minimized by incubating the sections in 2\% normal goat serum (Beyotime, Shanghai, China) in PBS for $20 \mathrm{~min}$. The sections were then incubated with anti-HPV58-E7 rabbit polyclonal antibody (1:500) at room temperature for 2 hours. Then, the sections were washed with PBS and incubated with an HRP-conjugated goat anti-rabbit IgG secondary or goat anti-mouse antibody (1:1000, Beyotime, Shanghai, China). The immunoreactions were visualized by incubating the sections for 3 minutes in a $0.1 \%$ 3, 30-diaminobenzidine (DAB) solution and counterstained with hematoxylin for 8 mins. The stained cells were observed under a microscope (Olympus, Tokyo, Japan). 


\section{Immunohistochemical data analysis}

The staining intensities of HPV58 E7, Ki-67 and p16 were graded on a scale from 0 to $3+(1+$ : weak, $2+$ : moderate, and 3+: strong) [10]. In brief, the positive reaction for three antibodies was scored into four grades, according to the intensity of the staining: 0,1 , 2 and 3 . The percentages of positive cells were also scored into five categories: 0 (0\%), 1 (1-25\%), 2 $(26-50 \%), 3(51-75 \%)$ and $4(76-100 \%)$. The product of the intensity by percentage scores was used as the final score, which was categorized into four groups: 0 $(<1), 1+(1-3), 2+(3-5), 3+(>5)$. The immunostained specimens were independently evaluated by two blinded investigators. There was close agreement $(>90 \%)$ between the two investigators; in the case of any disagreement, final grading was determined by consensus.

\section{Statistical analysis}

Categorical data analysis was conducted using the $\chi^{2}$ test. Either the Student's t-test or Wilcoxon test was performed to determine the differences between groups. Spearman's correlation was used for the correlation analysis. Results were considered statistically significant at $p<0.05$. All statistical analyses were done with SPSS22.0.

\section{Results}

\section{Patient characteristics}

Patient demographic and disease characteristics are summarized in Table 1 . In total, 67 patients (25 cancer patients and $42 \mathrm{CIN}$ patients) were included in the study. The median age at the time of diagnosis was 43 years old (range 27-65). Among 25 cervical cancer patients, 21 patients $(84 \%)$ were in cervical cancer stage $1,1(4 \%)$ in stage $2,2(8 \%)$ in stage 3 and 1 $(4 \%)$ in stage 4 . Of 42 CIN patients, $4(9.5 \%)$ were diagnosed as CIN1, $14(33.3 \%)$ were diagnosed as CIN2, and $24(57.1 \%)$ were diagnosed as CIN3. The median follow-up time was 41 months (range 2-240). It showed two cases of relapse, and two cases of death.

\section{Immunohistochemical results}

The results of immunohistochemical tests for HPV58 E7, p16 and Ki-67 are presented in Table 2. The expression of the HPV58 E7 protein was confirmed in $96 \%$ of the HPV58 positive cervical cancer tissues examined, and in $85.7 \%$ of HPV58 positive CIN tissues. The intensity of E7 staining was weak in 36\% of cancer cases, moderate in $28 \%$ of cancer cases and strong in $32 \%$ of cancer cases. The intensity of E7 staining was weak in $42.9 \%$ of CIN cases, moderate in $23.8 \%$ of CIN cases and strong in $19.0 \%$ of CIN cases.
P16 expression was found in $96 \%$ of the cancer group, and $97.6 \%$ of the CIN group. The intensity of p16 staining was weak in $4 \%$ of cancer cases, moderate in $4 \%$ of cancer cases and strong in $88 \%$ of cancer cases. The intensity of p16 staining was weak in $26.2 \%$ of CIN cases, moderate in $38.1 \%$ of CIN cases and strong in $33.3 \%$ of CIN cases. The expression of the Ki-67 protein was demonstrated in $96 \%$ cancer cases, and $83.3 \%$ CIN cases. The intensity of Ki-67 staining was weak in $0 \%$ of cancer cases, moderate in $28 \%$ of cancer cases and strong in $68 \%$ of cancer cases. The intensity of Ki-67 staining was weak in $7.1 \%$ of CIN cases, moderate in $54.3 \%$ of CIN cases and strong in $11.9 \%$ of CIN cases. As shown in Table 2, the expression level of HPV58 E7, p16, and Ki-67 in cervical cancer tissues were higher than in CIN tissues. Representative photographs of positive color reactions are shown in Figure 1 and Figure 2.

Table 1. Summary of patient demographic and disease characteristics in 67 patients

\begin{tabular}{ll}
\hline Characteristics & No. of pts \\
\hline Gender (female) & 67 \\
Age & $43(27-72)$ \\
Follow-up duration (months, median, range) & $41(2-240)$ \\
Staging of tumor* & \\
Pre-cancer & \\
Stage I & 42 \\
Stage 2 & 21 \\
Stage 3 & 1 \\
Stage 4 & 2 \\
Differentiation & 1 \\
Low & 16 \\
Intermediate & 8 \\
High & 1 \\
Unknown & 42 \\
Lymph node metastasis & \\
Yes & 3 \\
No & 64 \\
Diameter of tumor & \\
$<1$ cm & 57 \\
1-3 cm & \\
$>3 \mathrm{~cm}$ & 5 \\
Relapse & 5 \\
Yes & \\
No & \\
\hline Ninternatonal & \\
\hline
\end{tabular}

*International Federation of Gynecology and Obstetrics (FIGO) staging of carcinoma of the cervix uteri (2018).

Correlations between HPV58 E7, p16, or Ki-67 expression levels and clinical and pathological variables

Base on Table 2, we subdivided the staining results into 2 groups for further analyses: group 1 (weak and moderate staining), group 2 (strong staining). When we analyzed the age distribution of cervical cancer patients (Table 3), we found that HPV58 E7 expression level increased and Ki-67 expression decreased in older women (Table 4, 
$p<0.05)$. Correlations of HPV58 E7, p16 and Ki-67 expression levels with clinical and histological variables in cervical cancer patients were demonstrated in Table 4 . There were no significant differences in clinicopathological factors, including the degree of differentiation, the diameter of tumor lesions, staging of the tumor, lymph node metastasis, relapse, age of menarche, age of marriage, times of fetation and times of abortion between patients with low-moderate and strong expression level of HPV58 E7, p16, and Ki-67. However, p16 expression was associated with HPV58 E7 and Ki-67 expression (Table 4, $p<0.05$ ). As revealed by Spearman's correlation, there's a positive correlation between $\mathrm{p} 16$ expression and HPV58 E7 ( $\mathrm{r}=0.391, p=0.027)$ or Ki-67 expression $(\mathrm{r}=0.370, p=0.034)$. In CIN patients, there were no significant differences in clinicopathological factors between group 1 and group 2 .
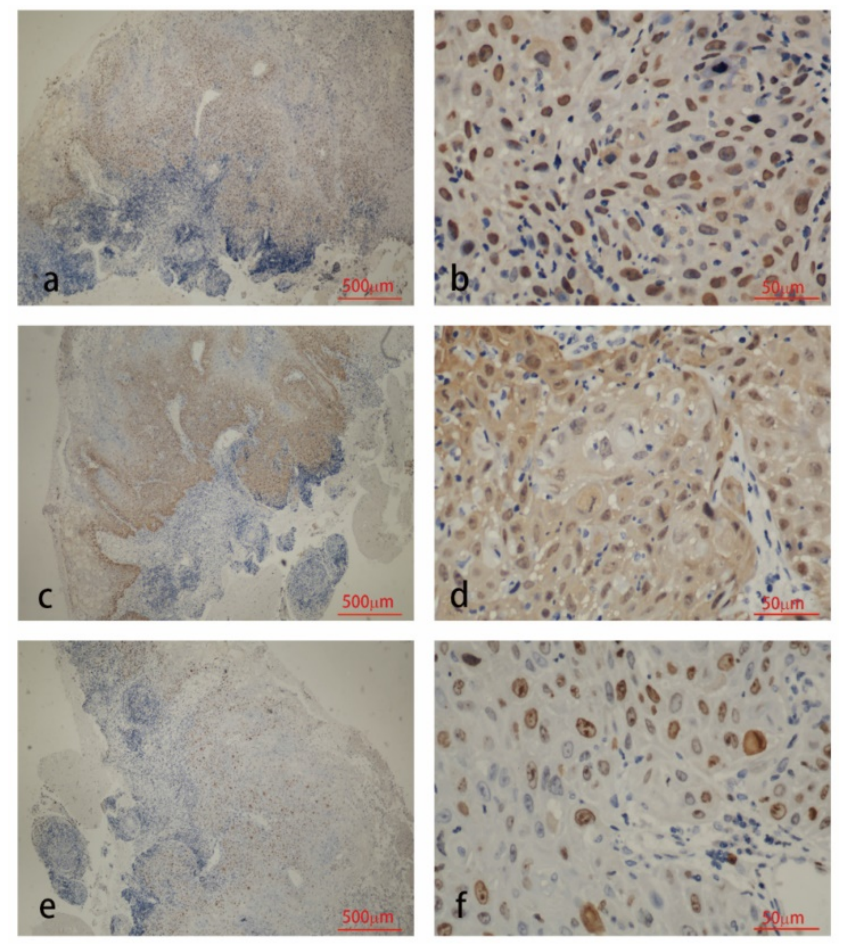

Figure 1. Immunohistochemistry staining for HPV58 E7 (a:×40, b:×400), p16 (c:×40, $\mathrm{d}: \times 400)$ and $\mathrm{Ki67}(\mathrm{e}: \times 40, \mathrm{f}: \times 400)$ in cervical cancer biopsies.

\section{Discussion}

In this study, we demonstrated the HPV58 E7 oncoprotein expression pattern in cervical cancer and $\mathrm{CIN}$ with immunohistochemistry. We determined the association of HPV58 E7 with other clinical and molecular parameters in cervical cancer samples.

Previous studies demonstrated that HR-HPV infection is associated with a broad spectrum of carcinogenesis in a variety of anatomic locations including the uterine cervix, vulva, anus, penis, and head and neck [11, 12]. The HPV 16, 18 types are mostly associated with cervical carcinoma worldwide [13]. HPV-58 is a common subtype of HR-HPV, which accounts for a notable proportion of the cervical cancers in Asian countries. It has been identified in 11.5 to $28 \%$ of cervical carcinoma patients in China, $8 \%$ in Japan and 16\% in Korea [14]. HPV16, 58, and 52 were the three most dominant HPV genotypes in cervical intraepithelial lesions in Shanghai [15]. In another study, they demonstrated HPV58 is the third most common HPV type in Eastern Asia overall [16].

DNA-based PCR is widely used in analyzing clinical specimens for the presence of HPV infection. However, the detection of DNA only indicates the presence of HPV and the expression of oncogenes are associated with progression to invasive cancer [17]. The E6 and E7 genes are the main oncogenes of HR-HPVs [18]. There should be an overexpression of the E7 gene from the integrated HPV genome. Some studies demonstrated the presence of E7-specific antibodies in sera in connection with increased risk of cervical cancer that may be detected up to 5 years prior to diagnosis [19]. However, the E7 antibodies were detected infrequently which might result from the low level of the HPV E7 antibodies in human sera. Another study showed seroprevalence of HPV18 E7 antibodies were significantly higher in HPV18 DNA-positive cervical cancer cases than controls. However, apparent limitations were observed. Firstly, cross-reactivity was evident among closely related HPV types. Secondly, one-third of cervical cancer cases showed no detectable E6 or E7 antibodies in sera among cases positive for HPV DNA [20]. Thus, we detected the E7 protein level in tissues of cervical cancer and CIN patients with the self-prepared polyclonal antibody [9]. The results revealed HPV58 E7 expressed in $96 \%$ of the HPV58 positive cervical cancer tissues examined, in $85.7 \%$ of HPV58 positive CIN tissues. The positive rate of E7 immunohistochemistry is relatively high in cervical cancer and CIN tissues. The method is worthy of clinical practice. The protein expression might be a useful marker for disease progression. However, there are still no type-specific commercial antibodies targeting HPV58 E7 oncoprotein in clinical application. In the current study, we evaluated the HPV58 E7 protein expression levels in cervical cancer and CIN samples by immunohistochemistry using a specific polyclonal antibody produced and verified by our research group [9]. We found that HPV58 E7 protein is highly expressed in cervical cancer and CIN samples by immunohistochemistry using this antibody, which provides evidence to support the reliability and potential of this antibody in clinical usage. 
Table 2. Immunohistochemical staining for HPV58 E7, p16, and Ki-67 in cervical cancer and CIN cases

\begin{tabular}{|c|c|c|c|c|c|c|c|c|c|c|c|c|c|c|}
\hline & \multirow[t]{2}{*}{ Group } & \multirow[t]{2}{*}{ Total cases (n) } & \multicolumn{4}{|c|}{ HPV58 E7 expression level, n (\%) } & \multicolumn{4}{|c|}{ P16 expression level, n (\%) } & \multicolumn{4}{|c|}{ Ki-67 expression level, n (\%) } \\
\hline & & & 0 & $1+$ & $2+$ & $3+$ & 0 & $1+$ & $2+$ & $3+$ & 0 & $1+$ & $2+$ & $3+$ \\
\hline \multirow{5}{*}{$\begin{array}{l}\text { Cervical } \\
\text { cancer }\end{array}$} & Stage 1 & 21 & $1(4.8)$ & $6(28.6)$ & $7(33.3)$ & $7(33.3)$ & $1(4.8)$ & $1(4.8)$ & $1(4.8)$ & $18(85.7)$ & $2(9.5)$ & $0(0.0)$ & $5(23.8)$ & $14(66.7)$ \\
\hline & Stage 2 & 1 & $0(0.0)$ & $1(100.0)$ & $0(0.0)$ & $0(0.0)$ & $0(0.0)$ & $0(0.0)$ & $0(0.0)$ & $1(100.0)$ & $0(0.0)$ & $0(0.0)$ & $1(0.0)$ & $0(0.0)$ \\
\hline & Stage 3 & 2 & $0(0.0)$ & $2(100.0)$ & $0(0.0)$ & $0(0.0)$ & $0(0.0)$ & $0(0.0)$ & $0(0.0)$ & $2(100.0)$ & $0(0.0)$ & $0(0.0)$ & $0(0.0)$ & $2(100.0)$ \\
\hline & Stage 4 & 1 & $0(0.0)$ & $0(0.0)$ & $0(0.0)$ & $1(100.0)$ & $0(0.0)$ & $0(0.0)$ & $0(0.0)$ & $1(100.0)$ & $0(0.0)$ & $0(0.0)$ & $0(0.0)$ & $1(100.0)$ \\
\hline & Total & 25 & $1(4.0)$ & $9(36.0)$ & $7(28.0)$ & $8(32.0)$ & $1(4.0)$ & $1(4.0)$ & $1(4.0)$ & $22(88.0)$ & $1(4.0)$ & $0(0.0)$ & 7 (28.0) & $17(68.0)$ \\
\hline \multirow[t]{4}{*}{$\mathrm{CIN}$} & CIN1 & 4 & $0(0.0)$ & $2(50.0)$ & $1(25.0)$ & $1(25.0)$ & $1(25.0)$ & $1(25.0)$ & $1(25.0)$ & $1(25.0)$ & $1(25.0)$ & $0(0.0)$ & $3(75.0)$ & $0(0.0)$ \\
\hline & CIN 2 & 14 & $0(0.0)$ & $5(35.7)$ & $4(28.6)$ & 5 (35.7) & $0(0.0)$ & $2(14.3)$ & 5 (35.7) & $7(50.0)$ & $3(21.4)$ & $1(7.1)$ & $8(57.1)$ & $2(14.3)$ \\
\hline & CIN 3 & 24 & $6(25.0)$ & $11(45.8)$ & $5(20.8)$ & $2(8.3)$ & $0(0.0)$ & $8(33.3)$ & $10(41.7)$ & $6(25.0)$ & $3(12.5)$ & $2(8.3)$ & $16(66.7)$ & $3(12.5)$ \\
\hline & Total & 42 & $6(14.3)$ & $18(42.9)$ & $10(23.8)$ & $8(19.0)$ & $1(2.4)$ & $11(26.2)$ & $16(38.1)$ & $14(33.3)$ & 7 (16.7) & $3(7.1)$ & $27(64.3)$ & $5(11.9)$ \\
\hline
\end{tabular}
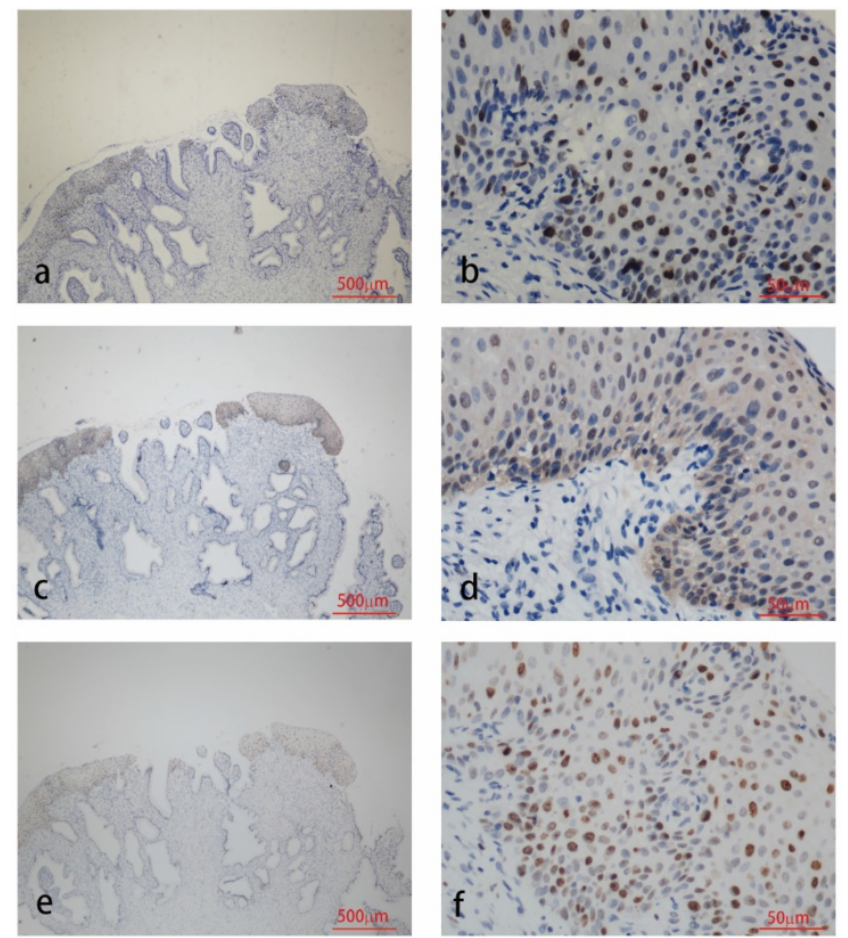

Figure 2. Immunohistochemistry staining for HPV58 E7 (a: $\times 40, \mathrm{~b}: \times 400)$, p16 (c: $\times 40$, $\mathrm{d}: \times 400)$ and $\mathrm{Ki67}(\mathrm{e}: \times 40, \mathrm{f}: \times 400)$ in $\mathrm{CIN}$ biopsies.

Table 3. Association between age and HPV58 E7, pl6 and Ki-67 staining

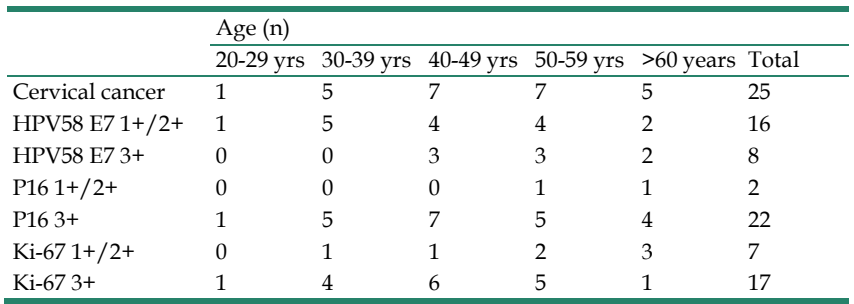

The HPV E7 protein in cooperation with E6 is essential for oncogenic cellular immortalization and transformation. E6 and E7 respectively induce the degradation of tumor suppressors p53 and retinoblastoma protein $\mathrm{pRb}[18,21]$. In addition, $\mathrm{HPV}$ E7 proteins associate with the pocket proteins, p107 and p130, via the Leu-X-Cys-X-Glu (LXCXE) motif in their conserved region (CR) 2 domain [22]. The pocket proteins regulate $\mathrm{G} 1 / \mathrm{S}$ transition by modulating the transcriptional activities of E2F transcription factors [23]. E2Fs also have effects on many other cellular processes relevant to tumorigenesis, including cellular differentiation, apoptosis, and genomic instability [24]. E7 proteins also interact with other proteins involved in cell proliferation, such as histone deacetylases [4], p21 and p27 cyclin-dependent kinase inhibitors [25] and components of the AP1 complex [26]. A report has indicated that HPV DNA replicates randomly or once per cell cycle in basal cells. The possible mechanism has been proposed, it may depend on the levels of the viral E1 replication protein and the cellular DNA replication protein components such as mini-chromosome-maintenance proteins (MCMs) [27]. A study showed HPV16-E7 transgenic keratinocytes with distinct cell surface markers attracted new antigen-presenting cell (APC) subsets to the epidermis. Transport of antigen to the draining lymph node by these APCs was markedly enhanced while antigen-processing was significantly impaired. They suggested E7 oncoprotein affected the phenotype and function of APCs and contributed to persisting infection with HPV [28]. HPV E7 may also induce the upregulation of RRM2 and promote ROS-ERK1/2-HIF-1a-VEGF-induced angiogenesis [29]. Furthermore, recent researches focused on immunotherapeutic approaches against E7-derived antigens in patients with cervical and oropharyngeal cancers [30, 31]. A study found that E6/E7 mRNA expression level was higher in women with high grade squamous intraepithelial lesion (HSIL) and CIN grade 2 or higher [32]. The combination of HPV-positive DNA and E6 or E7 serology could predict overall survival in individuals with HPV infections [33]. We found that CIN patients showed a lower rate of HPV58 E7 IHC positive compared to malignancy patients. The detection of the HPV E7 protein allows the monitoring of the oncogenic activity of the virus. 
Table 4. Clinical and histological correlations of immunohistochemical findings in cervical cancer ( $p$-value)

\begin{tabular}{|c|c|c|c|c|c|c|c|c|c|c|c|c|c|}
\hline & $\begin{array}{l}\text { HPV58 } \\
\text { E7 }\end{array}$ & P16 & Ki67 & $\begin{array}{l}\text { Diameter } \\
\text { of tumor }\end{array}$ & $\begin{array}{l}\text { Staging of } \\
\text { tumor }\end{array}$ & $\begin{array}{l}\text { Lymph node } \\
\text { metastasis }\end{array}$ & Relapse & Differentiation & Age & $\begin{array}{l}\text { Age of } \\
\text { menarche }\end{array}$ & $\begin{array}{l}\text { Age of } \\
\text { marriage }\end{array}$ & $\begin{array}{l}\text { Times of } \\
\text { fetation }\end{array}$ & $\begin{array}{l}\text { Times of } \\
\text { abortion }\end{array}$ \\
\hline HPV58E7 & - & 0.101 & 0.647 & 1.000 & 0.618 & 1.000 & 0.304 & 1.000 & $0.037^{*}$ & 0.426 & 0.629 & 0.582 & 1.000 \\
\hline P16 & $0.025^{*}$ & - & $0.025^{*}$ & 0.670 & 0.858 & 1.000 & 0.170 & 1.000 & 0.140 & 0.415 & 0.894 & 1.000 & 0.293 \\
\hline Ki-67 & 0.647 & 0.507 & - & 1.000 & 0.552 & 0.530 & 0.304 & 1.000 & $0.034^{*}$ & 0.635 & 0.470 & 0.098 & 0.108 \\
\hline
\end{tabular}

${ }^{*} p<0.05$.

The Ki-67 protein is a proliferation marker that is detected in the cell nuclei during the G1, S and G2 phases of the cell cycle and in mitosis. It has been elucidated that the protein is essential for the proliferation process [34]. The p16 ${ }^{\text {INK4a }}$, a cyclin-dependent kinase (CDK) inhibitor, decelerates the cell cycle by inactivating the CDKs that phosphorylates the retinoblastoma protein (RB) [35]. The expression of E6 and E7 proteins leads to p16 upregulation through the RB-p16 pathway. Thus, p16 could be a useful biomarker to improve the diagnosis of cervical cancer and CIN in combining with conventional morphology [36, 37]. The rates for p16 and $\mathrm{Ki}-67$ expression correlate with the severity of cervical neoplasia [38-40]. In this study, p16 expression was found in $96 \%$ of the cancer group, and in $97.6 \%$ of the CIN group. The expression of the Ki-67 protein was demonstrated in $96 \%$ cancer cases, and in 83.3\% CIN cases.

There were some limitations in our study. First, a smaller sample will give a result that may not be sufficiently powered to extrapolate the statistical difference. Second, as most of the cases were early-stage cervical cancers, survival analysis couldn't be conducted.

\section{Conclusions}

In conclusion, IHC for HPV58 E7 is a test with high specificity and sensitivity. The HPV58 E7 protein is highly expressed in cervical cancer and CIN patients. A novel marker for cervical cancer and CIN diagnosis and disease progression allows further implications for disease monitor. Larger studies are warranted to confirm our findings and to clarify the relationship between HPV58 E7 expression with clinical and pathological variables, which exhibits close correlations with other clinical and molecular parameters in cervical neoplasia, including p16 and Ki-67 expression. These findings provide evidence to support the reliability and potential of this antibody in clinical application [9].

\section{Abbreviations}

HR-HPV: high-risk human papillomavirus; CIN: cervical intraepithelial neoplasia; IHC: immunochemistry; FFPE: Formalin-fixed and paraffin- embedded; LEEP: Loop Electrosurgical Excision Procedure; PCR: polymerase chain reaction; DAB: diaminobenzidine; LXCX: E Leu-X-Cys-X-Glu; CR: conserved region; MCMs: mini-chromosomemaintenance proteins; APC: antigen-presenting cell; HSIL: high grade squamous intraepithelial lesion; CDK: cyclin-dependent kinase; RB: retinoblastoma protein; FIGO: International Federation of Gynecology and Obstetrics.

\section{Acknowledgements}

\section{Ethics Committee Approval and Patient Consent}

All procedures performed in studies involving human participants were in accordance with the ethical standards of Ethics Committee of Sir Run Run Shaw Hospital of Zhejiang University School of Medicine, China, and with the 1964 Helsinki declaration and its later amendments or comparable ethical standards. Informed consent was obtained from all individual participants included in the study.

\section{Funding}

This work was supported by the Science and Technology Projects of Zhejiang Province (2018C04013), the Research Foundation of Education Bureau of Zhejiang Province (No. Y201534631), Young Scientists Fund of the National Natural Science Foundation of China (No. 81801992), and the Medical and Health Science and Technology Project of Zhejiang Province (No. 2018256428).

\section{Authors' contributions}

Conception and design: HC, NJ. Development of methodology: QZ, XC, JZ, HC, NJ. Acquisition of data: QZ, XC, RH, LC, HW, HC. Analysis and interpretation of data: QZ, LC, JZ, YS, HC. Writing, review and/or revision of the manuscript: $\mathrm{QZ}, \mathrm{XC}$, $\mathrm{RH}, \mathrm{HC}, \mathrm{NJ}$. Administrative, technical, or material support: QZ, HW, LC, YS, HC, NJ. Study supervision: HC, NJ. All authors read and approved the final manuscript.

\section{Competing Interests}

The authors have declared that no competing interest exists. 


\section{References}

1. Bray F, Ferlay J, Soerjomataram I, et al. Global cancer statistics 2018: GLOBOCAN estimates of incidence and mortality worldwide for 36 cancers in 185 countries. CA Cancer J Clin. 2018; 68: 394-424.

2. de Martel C, Plummer M, Vignat J, et al. Worldwide burden of cancer attributable to HPV by site, country and HPV type. Int J Cancer. 2017; 141: 664-70.

3. Ding T, Wang X, Ye F, et al. Distribution of human papillomavirus 58 and 52 E6/E7 variants in cervical neoplasia in Chinese women. Gynecol Oncol. 2010; 119: 436-43.

4. Longworth MS, Laimins LA. Pathogenesis of human papillomaviruses in differentiating epithelia. Microbiol Mol Biol Rev. 2004; 68: 362-72.

5. Pal A, Kundu R. Human Papillomavirus E6 and E7: The Cervical Cancer Hallmarks and Targets for Therapy. Front Microbiol. 2019; 10: 3116.

6. Wise-Draper TM, Wells SI. Papillomavirus E6 and E7 proteins and their cellular targets. Front Biosci. 2008; 13: 1003-17.

7. Li $\mathrm{Y}$, Wang $\mathrm{X}, \mathrm{Ni} \mathrm{T}$, et al. Human papillomavirus type 58 genome variations and RNA expression in cervical lesions. J Virol. 2013; 87: 9313-22.

8. Chan PK, Zhang C, Park JS, et al. Geographical distribution and oncogenic risk association of human papillomavirus type $58 \mathrm{E} 6$ and E7 sequence variations. Int J Cancer. 2013; 132: 2528-36.

9. Zheng Q, Wang T, Jiang S, et al. Production of Polyclonal Antibody to the HPV58 E7 Protein and Its Detection in Cervical Cancer. PLoS One. 2016; 11: e0169138.

10. Ma Y, Ma L, Guo Q, et al. Expression of bone morphogenetic protein-2 and its receptors in epithelial ovarian cancer and their influence on the prognosis of ovarian cancer patients. J Exp Clin Cancer Res. 2010; 29: 85.

11. Darragh TM, Colgan TJ, Thomas Cox J, et al. The Lower Anogenital Squamous Terminology Standardization project for HPV-associated lesions: background and consensus recommendations from the College of American Pathologists and the American Society for Colposcopy and Cervical Pathology. Int J Gynecol Pathol. 2013; 32: 76-115.

12. Adelstein DJ, Ridge JA, Gillison ML, et al. Head and neck squamous cell cancer and the human papillomavirus: summary of a National Cancer Institute State of the Science Meeting, November 9-10, 2008, Washington, D.C. Head Neck. 2009; 31: 1393-422.

13. zur Hausen H. Papillomaviruses and cancer: from basic studies to clinical application. Nat Rev Cancer. 2002; 2: 342-50.

14. Chan PK. Human papillomavirus type 58: the unique role in cervical cancers in East Asia. Cell Biosci. 2012; 2: 17.

15. Gu Y, Ma C, Zou J, et al. Prevalence characteristics of high-risk human papillomaviruses in women living in Shanghai with cervical precancerous lesions and cancer. Oncotarget. 2016; 7: 24656-63.

16. Bao YP, Li N, Smith JS, et al. Human papillomavirus type distribution in women from Asia: a meta-analysis. Int J Gynecol Cancer. 2008; 18: 71-9.

17. Lee EY, Muller WJ. Oncogenes and tumor suppressor genes. Cold Spring Harb Perspect Biol. 2010; 2: a003236.

18. Fan X, Chen JJ. Regulation of cell cycle progression and apoptosis by the papillomavirus E6 oncogene. Crit Rev Eukaryot Gene Expr. 2004; 14: 183-202.

19. Lehtinen M, Pawlita M, Zumbach K, et al. Evaluation of antibody response to human papillomavirus early proteins in women in whom cervical cancer developed 1 to 20 years later. Am J Obstet Gynecol. 2003; 188: 49-55.

20. Combes JD, Pawlita M, Waterboer T, et al. Antibodies against high-risk human papillomavirus proteins as markers for invasive cervical cancer. Int J Cancer. 2014; 135: 2453-61

21. Hoppe-Seyler K, Bossler F, Braun JA, et al. The HPV E6/E7 Oncogenes: Key Factors for Viral Carcinogenesis and Therapeutic Targets. Trends Microbiol. 2018; 26: 158-68.

22. Dick FA, Dyson NJ. Three regions of the pRB pocket domain affect its inactivation by human papillomavirus E7 proteins. J Virol. 2002; 76: 6224-34.

23. Swiss VA, Casaccia P. Cell-context specific role of the $\mathrm{E} 2 \mathrm{~F} / \mathrm{Rb}$ pathway in development and disease. Glia. 2010; 58: 377-90.

24. Sage J. The retinoblastoma tumor suppressor and stem cell biology. Genes Dev. 2012; 26: 1409-20.

25. Funk JO, Waga S, Harry JB, et al. Inhibition of CDK activity and PCNA-dependent DNA replication by p21 is blocked by interaction with the HPV-16 E7 oncoprotein. Genes Dev. 1997; 11: 2090-100.

26. Antinore MJ, Birrer MJ, Patel D, et al. The human papillomavirus type 16 E7 gene product interacts with and trans-activates the AP1 family of transcription factors. EMBO J. 1996; 15: 1950-60.

27. Hoffmann $R$, Hirt B, Bechtold $V$, et al. Different modes of human papillomavirus DNA replication during maintenance. J Virol. 2006; 80: 4431-9.

28. Chandra J, Miao Y, Romoff N, et al. Epithelium Expressing the E7 Oncoprotein of HPV16 Attracts Immune-Modulatory Dendritic Cells to the Skin and Suppresses Their Antigen-Processing Capacity. PLoS One. 2016; 11: e0152886.

29. Wang $N$, Zhan $T, K e T$, et al. Increased expression of RRM2 by human papillomavirus E7 oncoprotein promotes angiogenesis in cervical cancer. Br J Cancer. 2014; 110: 1034-44.

30. Stern PL, van der Burg SH, Hampson IN, et al. Therapy of human papillomavirus-related disease. Vaccine. 2012; 30 Suppl 5: F71-82.

31. Nizard M, Sandoval F, Badoual C, et al. Immunotherapy of HPV-associated head and neck cancer: Critical parameters. Oncoimmunology. 2013; 2: e24534.
32. Valenca JE, Goncalves AK, Guerreiro da Silva ID, et al. High Risk HPV E6/E7 Oncoprotein Expression in Women with High Grade Squamous Intraepithelial Lesion. Rev Bras Ginecol Obstet. 2016; 38: 154-9.

33. Liang C, Marsit CJ, McClean MD, et al. Biomarkers of HPV in head and neck squamous cell carcinoma. Cancer Res. 2012; 72: 5004-13.

34. Scholzen T, Gerdes J. The Ki-67 protein: from the known and the unknown. J Cell Physiol. 2000; 182: 311-22.

35. Keating JT, Cviko A, Riethdorf S, et al. Ki-67, cyclin E, and p16INK4 are complimentary surrogate biomarkers for human papilloma virus-related cervical neoplasia. Am J Surg Pathol. 2001; 25: 884-91.

36. Benevolo $\mathrm{M}$, Mottolese $\mathrm{M}$, Marandino $\mathrm{F}$, et al. Immunohistochemical expression of p16(INK4a) is predictive of HR-HPV infection in cervical low-grade lesions. Mod Pathol. 2006; 19: 384-91.

37. Castle PE, Adcock R, Cuzick J, et al. Relationships of p16 Immunohistochemistry and Other Biomarkers With Diagnoses of Cervical Abnormalities: Implications for LAST Terminology. Arch Pathol Lab Med. 2020; 144: 725-34.

38. Kanthiya K, Khunnarong J, Tangjitgamol S, et al. Expression of the p16 and Ki67 in Cervical Squamous Intraepithelial Lesions and Cancer. Asian Pac J Cancer Prev. 2016; 17: 3201-6.

39. Toliman PJ, Phillips S, de Jong S, et al. Evaluation of p16/Ki-67 dual-stain cytology performed on self-collected vaginal and clinician-collected cervical specimens for the detection of cervical pre-cancer. Clin Microbiol Infect. 2020; 26: 748-52.

40. Shi Q, Xu L, Yang R, et al. Ki-67 and P16 proteins in cervical cancer and precancerous lesions of young women and the diagnostic value for cervical cancer and precancerous lesions. Oncol Lett. 2019; 18: 1351-5. 